\title{
Le pompage d'eau brute pour la réfrigération des centrales énergétiques et des complexes industriels
}

\author{
P. Courcot, G. Goudy, J. F. Lapray
}

GEC ALSTHOM BERGERON

Le bon fonctionnement des grands complexes industriels ou des centrales de production d'énergie est tributaire de la station de pompage qui les alimente, donc de sa sûreté de fonctionnement.

Les caractéristiques de l'eau et l'importance des débits de réfrigération sont les paramètres de base sur lesquels repose la conception de ces stations, des ouvrages de génie civil et des équipements électromécaniques.

Les pompes qui rentrent dans les stations de pompage de ces installations ont les caractéristiques suivantes:

- Complexes industriels (en pétrole, gaz, pétrochimie, GNL : Liquéfaction ou gazéification)

Débit unitaire de 4000 à $25000 \mathrm{~m}^{3} / \mathrm{h}$ pour des refoulements de 20 à $80 \mathrm{~m}$

- Centrales de production d'énergie.

- Centrales thermiques (de 250 à $600 \mathrm{MW}$ )

Débit unitaire de 15000 à $40000 \mathrm{~m}^{3} / \mathrm{h}$ pour des refoulements de 7 à $25 \mathrm{~m}$.

- Centrales thermiques à cycles combinés de 7000 à $22000 \mathrm{~m}^{3} / \mathrm{h}$ pour des refoulements de 7 à $25 \mathrm{~m}$.

- Centrales nucléaires

Réfrigération des condenseurs, débit unitaire de 60000 à $120000 \mathrm{~m}^{3} / \mathrm{h}$ pour des refoulements de 7 à $30 \mathrm{~m}$.

Trois types de pompes sont couramment installées pour ces circuits de refroidissements : ce sont des pompes verticales centrifuges :

— à corps immergé,

— à volute métallique,

— à volute en béton.

\section{LES POMPES}

\subsection{Choix du type de pompes}

Pour toutes ces applications, les pompes à axe vertical offrent un encombrement plus réduit et une liaison filtrepompe plus simple. Généralement les pompes de débit jusqu'à $50000 \mathrm{~m}^{3} / \mathrm{h}$ sont du type vertical à corps immergé (gamme SATURNE de GEC ALSTHOM BERGERON fig. l).

Toutefois, les pompes à volute métallique (fig. 2) s'imposent quand l'eau est très fortement chargée en sable. Pour des débits de 20000 à $50000 \mathrm{~m}^{3} / \mathrm{h}$, les pompes centrifuges ou hélico-centrifuges à volute en béton (fig. 3) viennent concurrencer les pompes verticales à corps im-

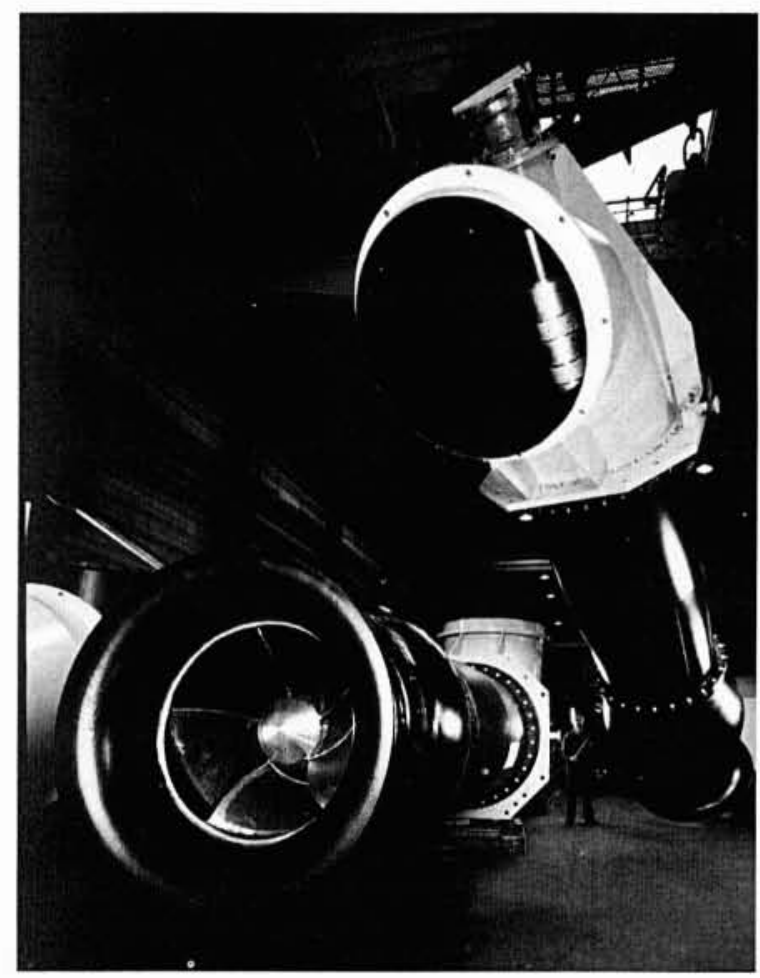

1. Pompes d'irrigation de Wadi El Nuqra (Egypte) : $23000 \mathrm{~m}^{3} / \mathrm{h}$ à $20 \mathrm{~m}-\mathrm{P}=1400 \mathrm{~kW}$.

mergé principalement dans les installations en eau de mer et pour les débits supérieurs, pour les raisons suivantes : - Les stators sont réalisés en matériaux résistants à la corrosion et la masse des pièces métalliques statoriques des pompes à corps immergés est supérieure à celle des pompes à volute béton.

- Les contraintes de maintenance conduisent les constructeurs à prévoir une conception « Pull out » (démontage rotor en laissant le stator en place).

Les débits supérieurs à $40000 \mathrm{~m}^{3} / \mathrm{h}$ constituent le domaine des pompes à volute en béton. Une conception pompe verticale à corps immergé, "Pull-out », avec fût en béton est concevable pour de tels débits.

1.2 Le dimensionnement hydraulique des pompes

Pour chacun des trois types considérés, le constructeur doit disposer d'une gamme de tracés hydrauliques performants 


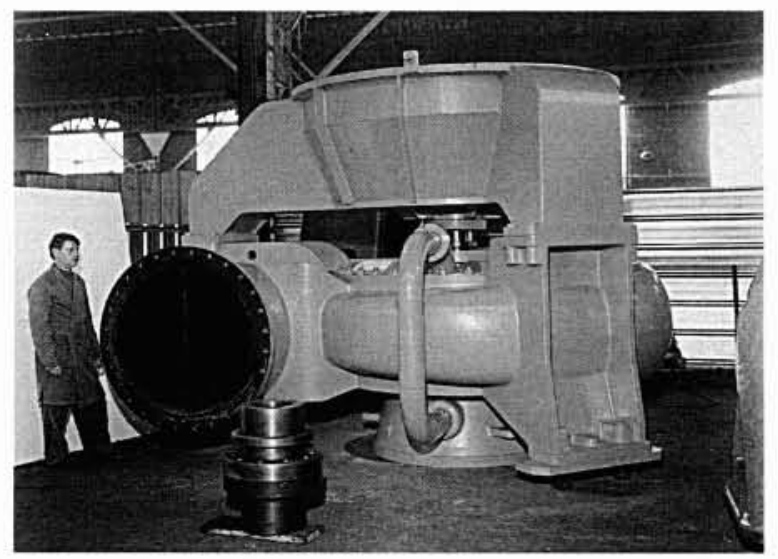

2. Pompe de refroidissement du complexe chimique Sonatrach-Annaba : $\quad 14500 \mathrm{~m}^{3} / \mathrm{h}$ à $\quad 40 \mathrm{~m}$. $P=2600 \mathrm{~kW}$.

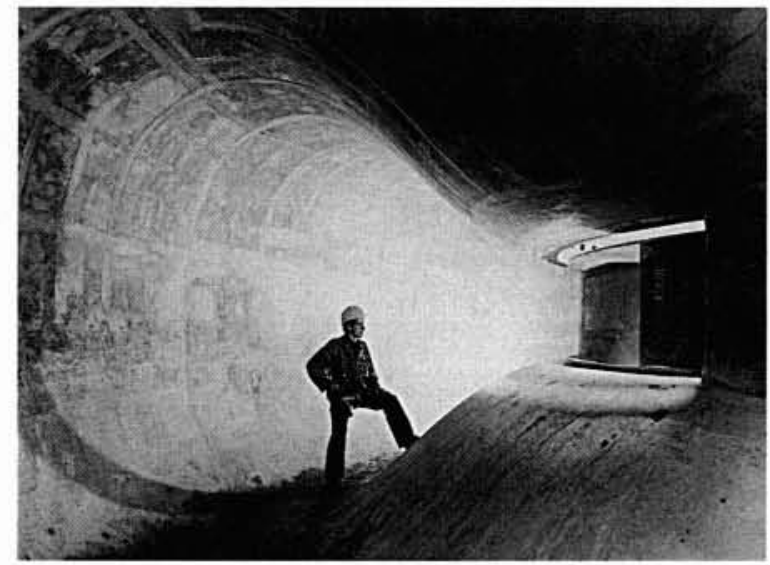

3. Pompe Volute Béton : intérieur de la volute.
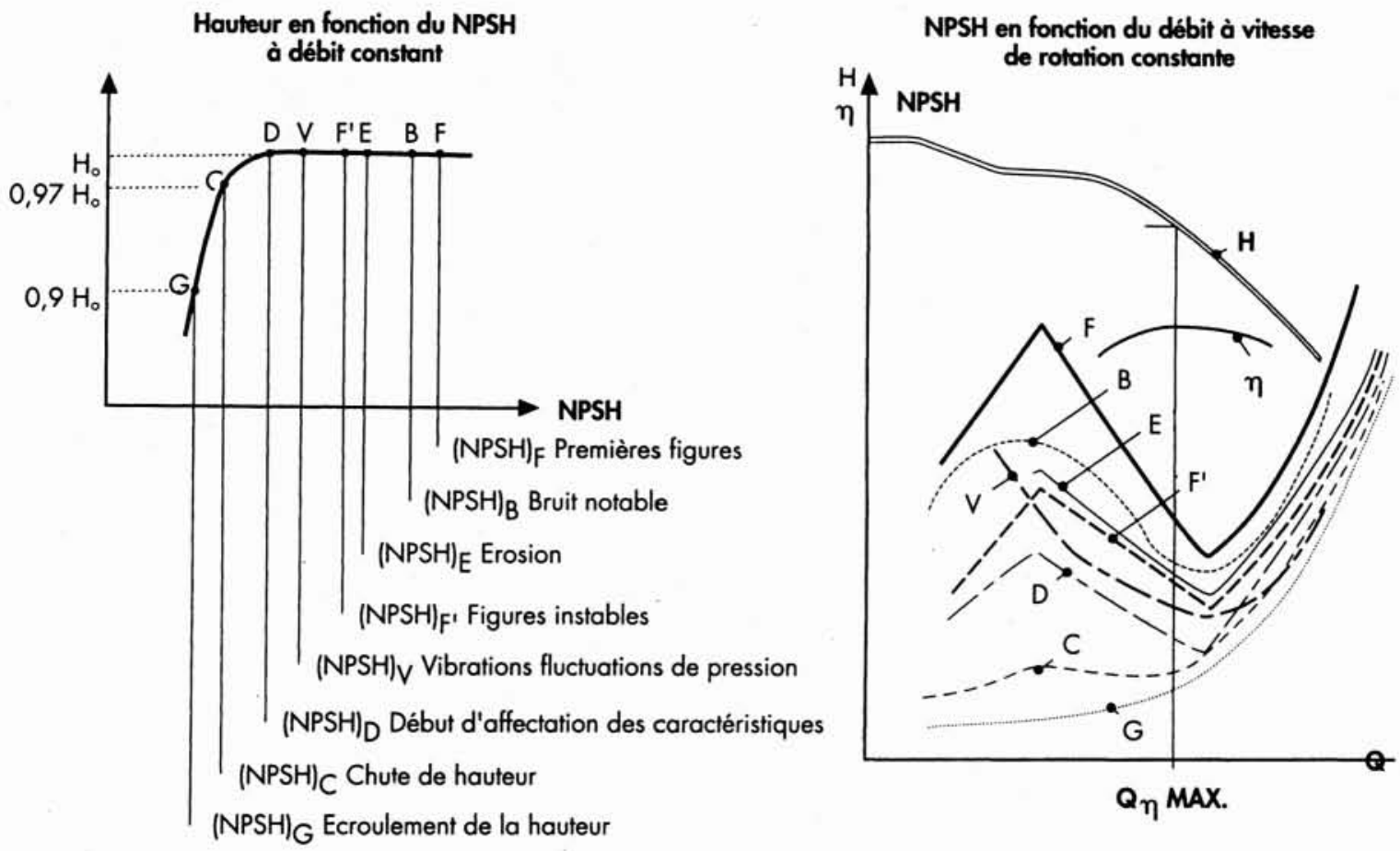

1.4 Les critères de NPSH et de vitesse spécifique d'aspiration

Les essais incluent systématiquement l'étude des capacités d'aspiration (NPSH) de la pompe (fig. 4).

Le travail expérimental comporte : la détermination des courbes de cavitation de la pompe $(\mathrm{NPSH})_{\mathrm{D}}$ ou $(\mathrm{NPSH})_{\mathrm{C}}$, ces valeurs correspondent respectivement au début d'affectation et à une chute arbitraire de $3 \%$ de la hauteur totale d'élévation.

La fiabilité du fonctionnement d'une pompe industrielle sera assurée lorsque le (NPSH) disponible fourni par l'ins-

Elles sont relevées au laboratoire sur des bouches d'essais rformantes.

ement étudié pour connaître avec certitude les zones d'uti-

ation des pompes industrielles.

connaître le comportement de la pompe en régimes transitoires anormaux et calculer les surpressions et dépressions dans les installations lors de disjonctions accidentelles.

dans toutes les zones d'utilisation. Les pompes industrielles sont extrapolées à partir de ces tracés de base en utilisant les lois de la similitude hydraulique.

\subsection{Les caractéristiques des pompes de base}

4. Courbes de NPSH. 
tallation à l'entrée de la pompe sera supérieur au (NPSH) requis par la pompe, dans la zone d'utilisation.

Ce critère peut parfois s'avérer insuffisant pour assurer une utilisation exempte de risques d'érosion de cavitation, de bruit, ou de vibration.

C'est pourquoi, il est préconisé de respecter une marge supplémentaire entre le (NPSH) disponible et le (NPSH) requis par la pompe, laquelle permet de couvrir, dans la zone de fonctionnement, les valeurs du (NPSH) requis par la pompe, correspondant à une figure de cavitation devenue instable et susceptible d'entraîner des risques d'érosion du fait des lâchers de cavités de vapeur dans l'écoulement: (NPHS) $)_{1}$.

Certaines normes ou standards imposent des limitations de la vitesse de rotation des pompes et de leurs vitesses spécifiques d'aspiration « $\mathrm{S}$ ».

$$
\left(S=\mathrm{Nrpm} \times Q^{0.5} \mathrm{~m}^{3} / \mathrm{s} \times \mathrm{NPSH}^{-0.75} \mathrm{~m}\right)
$$

Mais cette restriction, qui ne considère pas les critères expérimentaux décrits plus haut, n’assure pas toujours la fiabilité recherchée.

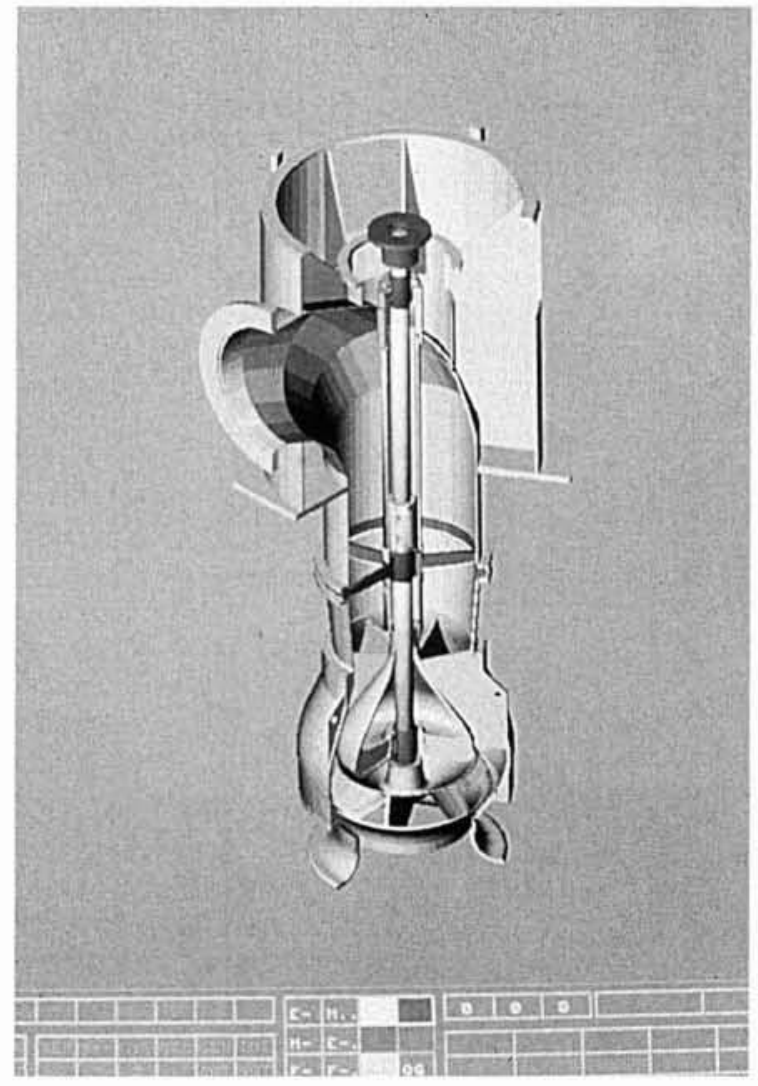

5. Pompe de refroidissement de la centrale de Kawas (Inde) : $11900 \mathrm{~m}^{3} / \mathrm{h}$ à $22,3 \mathrm{~m}-\mathrm{P}=840 \mathrm{~kW}$.

\subsection{Les validations sur pompe modèle réduit}

Certains contrats font obligation au Constructeur de valider leur pompe à l'aide d'un essai sur un modèle réduit, surtout lorsque la taille de la pompe industrielle empêche son essai sur site (cas des pompes à volute béton).

Ces essais incluent la modélisation d'une partie du conduit d'aspiration pour mieux cerner les problèmes de cavitation.

\section{POMPES VERTICALES À CORPS IM- MERGÉ}

Les pompes à corps immergé peuvent se classer en trois grandes familles :

- les pompes verticales métalliques avec des diamètres de refoulement de 150 à $2000 \mathrm{~mm}$,

- les pompes verticales métalliques type "Pull out" avec des diamètres de refoulement de 1000 à $2000 \mathrm{~mm}$,

- les pompes verticales «Pull out " à corps béton avec des diamètres de refoulements supérieurs à $1400 \mathrm{~mm}$.

Ces pompes sont constituées de sous-ensembles standardisés (fig. 5). On distingue principalement :

- le module hydraulique,

- la ligne d'arbre et les paliers de guidage,

- la colonne et le coude de refoulement,

- la boîte de butée (dans le cas où elle se situe sur la pompe),

- l'étanchéité de sortie d'arbre.

\subsection{Le module hydraulique}

Il assure la transformation de l'énergie mécanique en énergie hydraulique et comprend essentiellement

- la tulipe d'aspiration,

- la roue,

- le corps de pompe.

\subsection{La ligne d'arbre et les paliers de guidage}

La ligne d'arbre transmet le couple moteur à la roue. Elle est guidée par des paliers de façon à avoir la première vitesse critique de flexion supérieure à la vitesse de rotation.

Cette ligne d'arbre peut être réalisée en plusieurs tronçons.

Les paliers de guidage, noyés, sont généralement en caoutchouc, lubrifiés à l'eau et assurent le guidage de la ligne d'arbre avec des reprises d'efforts radiaux limités aux seules charges tournantes.

La forme du coussinet et l'épaisseur du caoutchouc assurent un bon comportement dynamique et un amortissement important.

En fonctionnement normal, la lubrification des coussinets caoutchouc est assurée soit directement par le fluide pompé, soit par un circuit extérieur d'eau propre filtrée.

Des chemises d'arbres sont prévues au droit des coussinets. Elles sont durcies soit par traitement thermique, ou par un revêtement de métallisation. 


\subsection{La colonne et le coude de refoulement}

Ils sont dimensionnés pour minimiser les pertes de charge entre la sortie de la cellule hydraulique et la bride de refoulement pompe.

\subsection{La boîte de butée}

La reprise de l'effort axial hydraulique généré par la roue ainsi que le poids du rotor pompe est assurée soit par une butée indépendante disposée sur la tête de pompe, soit par la propre butée du système d'entraînement (moteur ou réducteur).

\subsection{Le système d'étanchéité de sortie d'arbre}

Il est généralement du type à garniture à tresses compte tenu des faibles niveaux des pressions à étancher et des vitesses périphériques au droit du système d'étanchéité $(P<10$ bar ; $v<10 \mathrm{~m} / \mathrm{s})$.

Une chemise d'arbre durcie est prévue au droit des tresses pour limiter l'usure avec possibilité d'arrosage en eau propre. Des garnitures mécaniques peuvent être adaptées suivant la demande.

2.6 La conception de ces machines à partir des outils modernes

La conception modulaire des pompes verticales, permet de pouvoir s'adapter à de nombreuses contraintes d'installation (fig. 6)

Nos machines sont parfaitement adaptées à la conception par système C.A.O. adoptée pour GEC ALSTHOM BERGERON pour réduire les coûts et délais des études.

Des bases de données hydrauliques, technologiques et matériaux ont été constituées d'une part à partir d'études fonctionnelles menées sur le produit (ANAV, AMDEC) et d'autre part à partir du retour d'expérience.

La souplesse de l'outil permet de pouvoir répondre à toute demande spécifique. A partir d'un Cahier des Charges Fonctionnel d'une pompe entrant dans la gamme SATURNE, il est possible de définir complètement la machine, en quelques heures.

\section{POMPES À VOLUTE EN BÉTON (P.V.B)}

Ce type de pompe est couramment utilisé pour des débits unitaires de 18000 à $120000 \mathrm{~m}^{3} / \mathrm{h}$ avec hauteurs de 7 à $30 \mathrm{~m}$.

\subsection{La technologie}

La pompe à volute en béton diffère des pompes conventionnelles du fait que le conduit d'aspiration et la volute sont construits en béton armé et font parties intégrantes de la structure de la station de pompage.
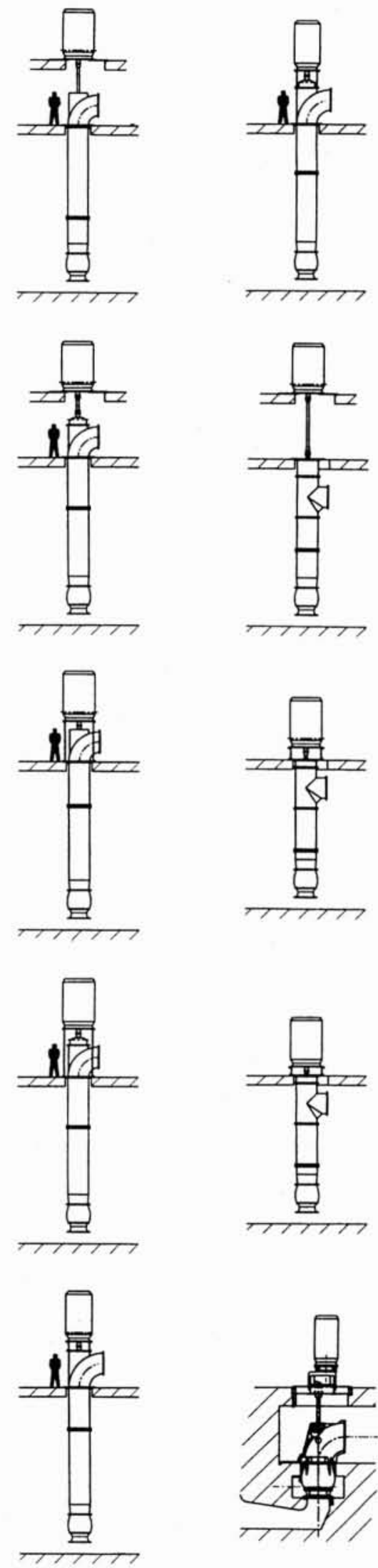

6. Arrangements divers des pompes verticales. 
Ce sont des pompes à axe vertical du type « Pull-out », les éléments internes constituant la pompe étant démontables en bloc du type cartouche.

\subsection{Eléments en béton}

On distingue :

- Le conduit d'aspiration

Il est inséré dans le Génie Civil de la station de pompage. Différentes formes peuvent être adoptées de façon à satisfaire aux contraintes de la section d'entrée (fig. 7). - La volute (fig. 8)

La forme de la volute est étudiée de façon à répondre à trois exigences :

— un rendement hydraulique élevé,

- un coffrage simple à mettre en œuvre,

- une mise en place du ferraillage et une coulée du béton aussi aisées que possible.

\subsection{Les pièces mécaniques scellées dans le béton}

\section{On distingue :}

— une pièce scellée à l'aspiration, servant à fixer la bague d'étanchéité,

- une pièce scellée en partie supérieure, destinée à recevoir le fond de corps de pompe,

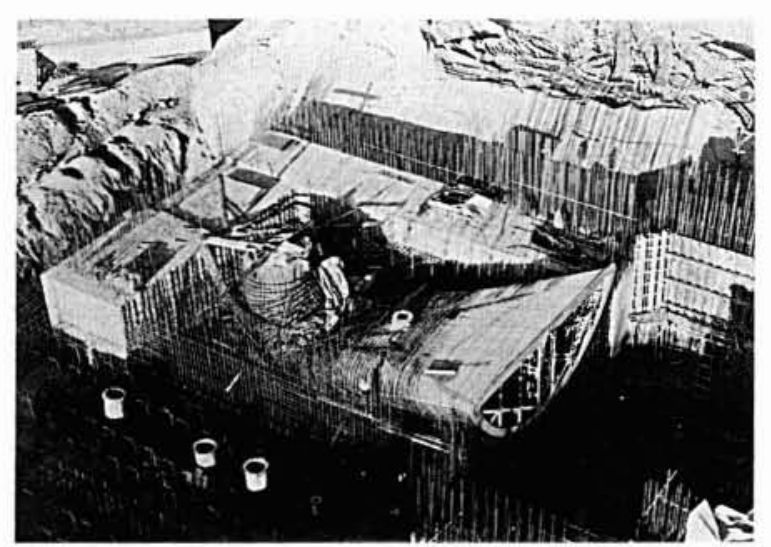

7. Coude de pompe Volute Béton KNUS (Corée du Sud) : $110000 \mathrm{~m}^{3} / \mathrm{h}$ à $15 \mathrm{~m}-\mathrm{P}=5000 \mathrm{~kW}$.

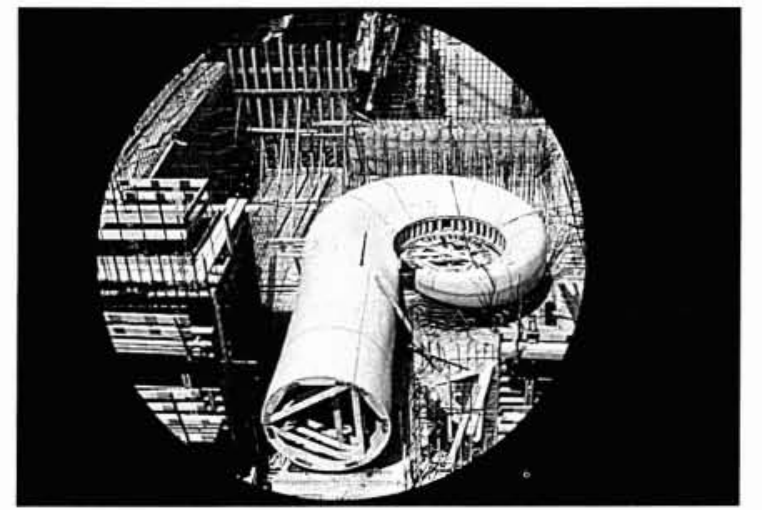

8. Coffrage de pompe volute béton de la centrale nucléaire de Paluel (France).
— une languette scellée, ou « bec de volute » qui protège localement le béton dans cette zone de la volute.

\subsection{Parties démontables}

On distingue :

- Les bagues d'usure

Sur la pièce d'aspiration, la bague d'usure (dite d'étanchéité) sert à limiter le débit de fuite provenant de la sortie roue vers l'aspiration.

- La roue (Fig. 9)

Elle est du type centrifuge ou hélico centrifuge selon la vitesse spécifique

$$
\left[\mathrm{ns}=N(\mathrm{rpm}) \cdot Q^{1 / 2}\left(\mathrm{~m}^{3} / \mathrm{s}\right) \cdot \mathrm{H}^{3 / 4}(\mathrm{~m})\right] .
$$

La roue centrifuge est utilisée pour des vitesses spécifiques comprises entre 70 et 100 et la roue hélico centrifuge pour des vitesses spécifiques variant de 90 à 150 .

\section{- L'arbre}

L'un des avantages des P.V.B est que l'arbre n'est jamais en contact direct avec le liquide pompé. Des chemises d'arbres démontables sont prévues à la partie basse sous le couvercle de la boîte de presse-étoupe.

- Les paliers

Différents types de paliers peuvent être utilisés avec un ou deux paliers-guide. L'une des solutions les plus fiables et de monter seulement un palier-cuve à huile tournante situé au-dessus du boîtier de l'étanchéité de sortie d'arbre. - La butée

Elle est conventionnelle soit à roulement soit à patins à huile et peut être installée sur le fond de corps de pompe, dans le réducteur de vitesse ou dans le moteur (pour les pompes entraînées directement par un moteur lent).

\section{- L'étanchéité d'arbre}

Dans la plupart des cas, l'étanchéité au passage de l'arbre est assurée au moyen d'un boîtier de presse-étoupe et les tresses sont refroidies par de l'eau de refroidissement prise directement au refoulement ou via un système de filtration.

\subsection{Construction et installation}

\section{- Coffrages}

Les coffrages sont composés d'éléments assemblés en bois, éventuellement réutilisables.

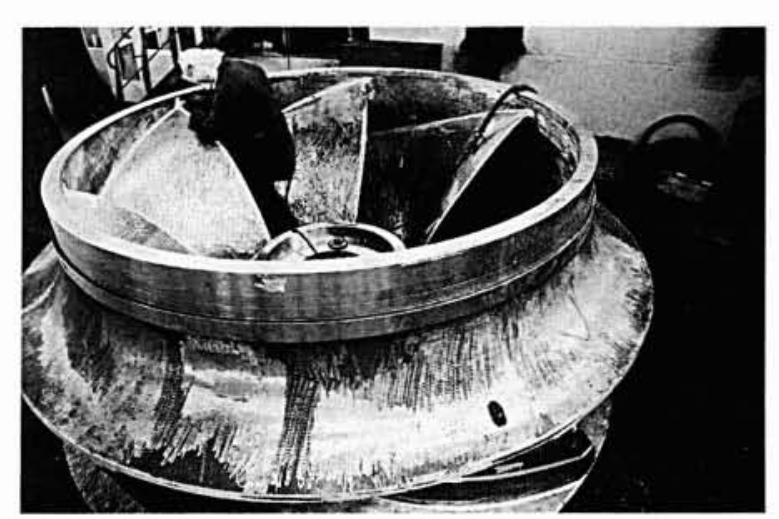

9. Roue de pompe Volute Béton de la centrale nucléaire de KNU9 (Corée du Sud). 
Le béton doit être de qualité normale, et généralement aucun traitement de surface n'est nécessaire.

\section{- Les pièces à sceller}

Elles sont maintenues en position à l'aide d'un dispositif qui permet de couler le béton très rapidement. Le montage et l'alignement des autres pièces principales dépendent de la qualité du calage mécanique de ces pièces scellées.

\subsection{Architecture générale des groupes de pompage}

Les installations sont très souples et doivent s'adapter aux contraintes variables d'un site à l'autre.

L'entraînement peut être effectué par moteur rapide couplé à un réducteur, soit par moteur lent. Cette seconde solution est souvent jugée plus fiable.

\subsection{Principaux avantages des pompes à volute en béton}

- elles offrent une solution technico-économique très compétitive pour des débits d'eau importants ;

- la volute et le conduit d'aspiration sont extrêmement résistants, plus particulièrement en eau de mer ;

- la masse et le coût des parties métalliques sont faibles ; - la volute en béton et le conduit d'aspiration sont faciles à mettre en cuvre ;

- le palier est hors d'eau, ce qui entraîne une usure très réduite, une conduite et une maintenance très simplifiées ; - excepté la chemise de protection en partie basse, l'arbre n'est jamais en contact avec l'eau,

- les vitesses de rotation sont lentes; la roue peut-être équilibrée hydrauliquement ;

- la roue peut-être inspectée sans démontage de la pompe et sans mise hors d'eau de l'installation;

- les moyens de manutention et les hauteurs sous crochet sont réduits ;

- comme en pompe « Pull-out », l'arbre et la roue peuvent être retirés rapidement ;
- la conception simple et éprouvée confère une fiabilité exceptionnelle avec une maintenance préventive annuelle et un démontage tous les cinq ou dix ans ;

- le dessin hydraulique de l'ensemble permet des rendements globaux très élevés (souvent supérieurs à $90 \%$ ).

La très grande fiabilité des pompes à volute en béton a conduit à installer une seule pompe par tranche pour un grand nombre de centrales thermiques (systématique en France) et deux pompes par tranche pour les centrales 900 à $1400 \mathrm{MW}$ malgré les débits et la taille des machines (60 000 à $110000 \mathrm{~m}^{3} / \mathrm{h}$ par pompe).

\section{LES POMPES À VOLUTE MÉTALLI- QUE}

Ces pompes sont du type monocellulaire, en volute, à axe vertical. Elles sont constituées par :

- un corps de pompe métallique, du type volute,

— un ensemble cartouche démontable, qui comprend:

- la roue,

- l'arbre,

- le fond de corps avec le système d'étanchéité,

- le corps de palier avec un palier de guidage et la butée,

- le demi-manchon d'accouplement,

- un support-moteur,

- le moteur d'entraînement est disposé en chandelle sur la pompe.

Les avantages des pompes à volute métallique résident essentiellement dans le nombre limité des pièces en contact avec le fluide pompé.

Pour le corps de pompe, des fontes faiblement ou fortement alliées répondent aux sollicitations rencontrées. Ce choix technico-économique est intéressant quand certains corps de pompes peuvent atteindre 15 à 20 tonnes.

Tableau 1. - Choix des matériaux pour pompes en fonction de la qualité de l'eau.

\begin{tabular}{|l|l|l|l|}
\hline $\begin{array}{l}\text { Qualité de l'eau } \\
\text { Pièces }\end{array}$ & \multicolumn{1}{|c|}{$\begin{array}{c}\text { Eau douce } \\
\text { sans sable }\end{array}$} & \multicolumn{1}{|c|}{$\begin{array}{c}\text { Eau douce } \\
\text { avec sable }\end{array}$} & \multicolumn{1}{|c|}{$\begin{array}{c}\text { Eau de mer } \\
\text { non polluée }\end{array}$} \\
\hline Roue & Cupro aluminium & $\begin{array}{l}\text { à l'Acier inoxydable } \\
\text { martensitique }\end{array}$ & $\begin{array}{l}\text { Cupro aluminium Acier } \\
\text { inoxydable } \\
\text { austénitique ou } \\
\text { austéno-ferritique } \\
\text { (Duplex) }\end{array}$ \\
\hline $\begin{array}{l}\text { Arbre } \\
\text { a/ Pompe à volute (non } \\
\text { en contract avec l'eau) }\end{array}$ & Acier au carbone & Acier au carbone & $\begin{array}{l}\text { Acier inoxydable } \\
\text { martensitique }\end{array}$ \\
\hline $\begin{array}{l}\text { b/ Pompe verticale à } \\
\text { corps immergé }\end{array}$ & $\begin{array}{l}\text { Acier inoxydable } \\
\text { martensitique }\end{array}$ & $\begin{array}{l}\text { Acier inoxydable } \\
\text { martensitique }\end{array}$ & $\begin{array}{l}\text { Acier inoxydable } \\
\text { austénitique ou } \\
\text { austéno-ferritique } \\
\text { (Duplex) }\end{array}$ \\
\hline $\begin{array}{l}\text { Pièces statoriques en } \\
\text { contact avec l'eau }\end{array}$ & $\begin{array}{l}\text { Pièces moulées } \\
\text { en fonte non alliée }\end{array}$ & $\begin{array}{l}\text { Pièces moulées en fonte } \\
\text { faiblement alliée ou } \\
\text { aciers anti-abrasion }\end{array}$ & $\begin{array}{l}\text { Pièces moulées en fonte } \\
\text { Ni-Résist ou } \\
\text { Acier inoxydable } \\
\text { austénitique }\end{array}$ \\
\hline
\end{tabular}


Pour améliorer la tenue à la corrosion en eau de mer et/ou à l'abrasion, des revêtements spéciaux peuvent être effectués intérieurement et ce revêtement est facile à réaliser et à renouveler.

Pour les pièces les plus sollicitées, des dispositions particulières peuvent être mises en place pour résoudre les problèmes d'érosion par abrasion dans les cas difficiles.

La disposition de la butée dans le moteur permet d'avoir des groupes compacts et de supprimer un ensemble mécanique (butée pompe).

\section{V — CHOIX DES MATÉRIAUX}

Les matériaux couramment utilisés varient selon le composant mécanique et la nature de l'eau (tableau l).

\section{VI $\square$ CONCLUSION}

GEC ALSTHOM BERGERON est capable d'étudier et de réaliser tous les types de pompes adaptées aux stations de pompage, des grands complexes industriels et des centrales énergétiques et de construire ces pompes tant en France que dans des pays étrangers suivant le type de pompes et la nature du projet.

Pour de telles installations GEC ALSTHOM BERGERON possède la maîtrise de la réalisation de stations de pompage clefs en main, compte tenu de son expérience et de son savoir-faire en architecture hydraulique et en conception mécanique de machines de grandes dimensions. 